\title{
Collaborative Dynamic Optimization on Post-earthquake Emergency Sup- ply and Road Rehabilitation Based on Road Network Connectivity
}

\author{
He Shanshan ${ }^{1}$, Zhu Wenhai ${ }^{2}$ and Wang Chaofeng ${ }^{3, *}$ \\ ${ }^{1}$ Basic Department, Institute of Disaster Prevention, Sanhe 065201, China \\ ${ }^{2}$ Fengtai Railway Freight Center, Beijing Railway Administration, Beijing 102300, China \\ ${ }^{3}$ School of Airport Engineering \& Transportation Management, Civil Aviation Flight University of China, Guanghan \\ 618307, China
}

\begin{abstract}
Earthquakes often cause road damage, so the earthquake relief needs cooperative road rehabilitation and postearthquake emergency supply. In the thesis, the definition of connectivity degree on post-earthquake road network is proposed, and the rehabilitation order for road network is resolved according to its extended meaning. Then, the multistage dynamic problem is decomposed to multiple single phase problems. For each single one, the mathematical model, which balances between efficiency and fairness, is established, and a simulated annealing algorithm is adopted for solving the model, at last its feasibility is verified by a numerical example.
\end{abstract}

Keywords: Emergency supply, road network connectivity, road rehabilitation.

\section{INTRODUCTION}

Earthquake is a common natural disaster. Due to the technical limitations of prediction and the occurrence of sudden, earthquake often cause huge losses of personal safety and property. After the earthquake, the disaster area has a large demand for food, water, tents, quilts and other materials, which to maintain the disaster area people's daily life and carry out relief work. Therefore, timely delivery of emergency supplies plays a vital role for the earthquake relief, saving lives, reducing other losses. At the same time, the earthquake can cause the road network damage in different degrees, which need synchronous carry out road rehabilitation work. During the period of earthquake disaster relief, on the one hand, the aftershocks and secondary disasters can damage the road again, on the other hand, partially damaged road repair work will be completed, so the connectivity for road network is in dynamic process. Because the road traffic conditions leading to the disaster area the directly affect the relief distribution path selection and distribution efficiency, and even affect the accessibility of relief supplies. It is of great significance to study the road rehabilitation and emergency supplies distribution in the dynamic road network based on connectivity.

Many domestic and foreign scholars from different angle to study the emergency supplies distribution [1-4] and post disaster road repair [5-8] problem. From the persoective of emergency material distribution, most scholars put the emergency vehicle scheduling are classified as a special kind of

*Address correspondence to this author at the School of Airport Engineering \& Transportation Management, Civil Aviation Flight University of China, Guanghan 618307, China; E-mail: chaofengbrad@126.com vehicle routing problem (VFP) [9], and carries out the relevant research. For the research of road rehabilitation, the order fro repair is focused on. In recent years, some scholars began to research on the integrated optimization of emergency supplies distribution and road repair problems, such as Shi Youlin [10], Zhang Yi [11], Li Aiqing [12], who established multi-objective mixed integer models using the repair time and distribution time as the goal and developed effective heuristics. Liao [13] respectively maximization of road rehabilitation performance and minization of delivery time as the target, established a bi-level programming model of post-earthquake road rehabilitation and emergency supply. In addition, there are some other literatures [14-16] on the integrated optimization. However, the present research is concerned, the road network repair order problem of less consideration of road network dynamic change and the connectivity.

In view of this, the paper established a mathematical model to describe the dynamic integration relation between post earthquake road rehabilitation and emergency material distribution, from the perspective of road network connectivity, thus obtained some valuable conclusion.

\section{PROBLEM DESCRIPTION AND NOTATIONS}

\subsection{Problem Description}

The road network and communication facilities were damaged to delay information transfer and acquisition, owing to the characteristics of earthquake emergency. The damaged roads need rehabilitation, which is the basis of emergency material distribution. The earthquake information in a dynamic change process, thanks to the road network and 
communication facilities repaired, and damaged again by aftershocks and secondary disasters. Each stage of information should be collected to provide dynamic information necessary to decision-making for disaster relief work. In summary, integrated optimization problem of road network rehabilitation and emergency supply can be regarded as a multi-stage dynamic process. The process can be transformed static process of every stage, and every network transformation (that is, every road damaged every road repair completed) is regarded as the time of the node for stage division. For the information transmission between multi-stages, one stage of unfinished work (such as the vehicle not to back to the rescue center, and relief materials not to meet the demands) is regarded as the initial information to the next stage, meanwhile, new generated information (such as relief demand distribution, and damage repair road) is considered to optimize the phase problem of emergency distribution and road rehabilitation.

\subsection{Basic Hypothesis}

Hypothesis 1: An emergency rescue center, responsible for the arrangements of post earthquake emergency material distribution and road rehabilitation, was set up. And the emergency rescue was considered as the starting point for distribution.

Hypothesis 2: Due to a large amount demand of relief supplies, direct distribution pattern was adopted. Vehicle started from the emergency rescue center, adopted the shortest path way to a certain demand point in the available network conditions, and returned to the emergency rescue center after discharge.

Hypothesis 3: In the entire rescue process, the total number of distribution vehicles is constant, and one vehicle is only responsible for one demand point repeated distribution.

Hypothesis 4: The capacity of emergency rescue center is not limited, because of the relief materials sually continuous to emergency rescue center.

\subsection{Notations}

$E_{o d}$ : The road network connectivity of post-earthquake, from the emergency rescue center $o$ to demand point $d$;

$E_{\text {od }}^{\prime}$ : The road network weighted connectivity of postearthquake, from the emergency rescue center $o$ to demand point $d$;

$D$ : The set of demand points;

$M$ : The set of emergency material kinds;

$K$ : The set of distribution vehicles;

$Q_{d m}$ : The quantity demanded of the demand point $d$ for the material kind $m$;

$A_{d m}:$ The cumulative distribution quantity of the demand point $d$ for material kind $m$; $w_{d}$ : Demand urgent coefficient of the demand point $d$, $\sum_{d \in D} w_{d}=1$

$S_{k d m}$ : The quantity of vehicle $k$ carrying the material kind $m$ to demand point $d$;

$\Delta S_{t d m}$ : The delayed distribution quantity of the demand point $d$ for the material kind $m$ and the delayed time $t$;

$t_{\text {od }}$ : The time of arriving the demand point $d$ from the emergency rescue center;

$t_{k d m}$ : The time of distribution vehicle $k$ carrying the material kind $m$ and arriving the demand point $d$;

$t_{d m}^{l}$ : The required deadline of the demand point $d$ for the material $m$;

$\Delta t_{k d m}$ : The delayed time of distribution vehicle $k$ carrying the material kind $m$ and arriving the demand point $d$; If $t \leq t_{d m}^{l}$, then $\Delta t_{k d m}=0$, else $\Delta t_{k i m}=i_{k d m}-t_{d m}^{l}$;

$N_{t d m}$ : The number of repeated distribution of vehicle $k$ carrying the material kind $m$ and arriving the demand point $d$;

$T_{i k}$ : The time of vehicle $k$ arriving the node $i$ of road network;

$\delta_{i j k}$ : The required time of vehicle $k$ passing by the road section $(i, j)$;

$n$ : The number of distribution vehicle;

$x_{i j k}$ : The 0-1 variable; If vehicle $k$ passing by the road section $(i, j)$, then setting to 1 , else 0 ;

$y_{\text {odk }}$ : The $0-1$ variable; If vehicle $k$ arriving the demand point $d$, then setting to 1 , else 0 ;

$\alpha$ 、 $\beta$ : The consistency adjustment coefficients;

\section{MODEL FORMULATION}

\subsection{Road Network Connectivity of Post-Earthquake}

Connectivity is a feature representation of road network structure, and reacts each node accessibility. After the earthquake, some road damaged to change the road network structure. Once one certain road was interrupted, the road network remaining would act as the lifeline role, to make the vehicles to rescue wounded and emergency material distribution. If the relief point could not be access by virtue of the remaining road network, the roadmust be rehabilitated priority. Therefore, which road need to be repair priority is a question for worthy considering, thus the concept of "post- earthquake road connectivity" is put forward. 


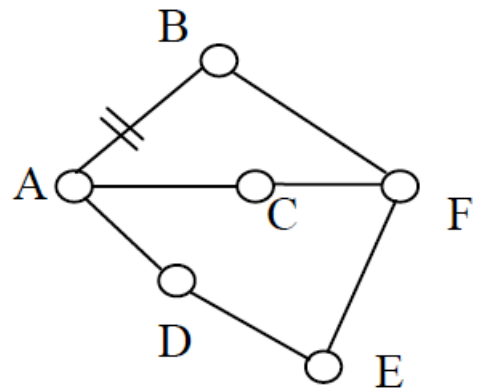

Fig. (1). Schematic diagram on concept of post-earthquake road section connectivity.

\subsubsection{Concept of Post-Earthquake Road Connectivity}

Definition: The earthquake causes some certain road interruption, and accessing one node belonging to the section depends on the remainder of the road path. It is defined the post-earthquake road section connectivity to the probability of connecting.

As shown in Fig. (1), the section AB is interrupted owing to the earthquake, thus the remaining paths $\mathrm{AB}$ are $\mathrm{AC}-\mathrm{CF}-$ FB and AD-DE-EF-FB. The two paths in the connectivity probability is defined as post-earthquake road section $\mathrm{AB}$ connectivity, denoted as $E_{A B}$.

In the post-earthquake road network, if one certain section is in the connection, the post-earthquake road section connectivity is 1 , else 0 .

$X_{i j}= \begin{cases}0 & \text { when the } \operatorname{section}(i, j) \text { is unconnected } \\ 1 & \text { when the } \operatorname{section}(i, j) \text { is connected }\end{cases}$

The sections connectivity can be expressed as expected value.

$E_{i j}=P\left(X_{i j}=1\right)$

In addition, the probability of sections unconnected is represented by the following formula.

$P\left(X_{i j}=0\right)=1-E_{i j}$
3.1.2. Connectivity of the Road Network of the Basic Structure

The network contains two kinds of basic structure of series and parallel. As shown in Fig. (2).

As shown in Fig. (2.1), in the series structure, $e_{i, j}$ represent the edge. Then the connectivity from $\mathrm{O}$ to $\mathrm{D}$ is represented by the following formula.

$$
E_{O D}=\prod_{e_{i j} \in R} E_{\mathrm{ij}}
$$

As shown in Fig. (2.1), in the series structure, $e_{l}$ represent the edge. Then the connectivity from $\mathrm{O}$ to $\mathrm{D}$ is represented by the following formula.

$$
E_{O D}=1-\prod_{e_{l} \in R}\left(1-E_{l}\right)
$$

\subsubsection{Connectivity of the Road Network of the General Structure}

General road network is composed of series and parallel connection of the basic structure. Usually the gen e ral road network can be decomposed into simple connected network structure. The whole path connectivity can be obtained through the analysis of related to the series structure according to the formula (3), a parallel structure based on the formula (4).

\subsection{Collaborative Dynamic Optimization Problem}

\subsubsection{Conbersion of Multi Stage Dynamic Problem}

After the earthquake, on the one hand, people's productions and lives are impacted, on the other hand, some road sections are interrupted. In this case, the road rehabilitation and emergency distribution need to Synchronous develop. The road network is in the dynamic change process due to rehabilitation and secondary disasters, repair section completed, and new section damaged. Due to the dynamic nature of road network, the post disaster distribution relied on is uncertain. Every change in road network is divided as a stage, thus the distribution optimization can be transformed

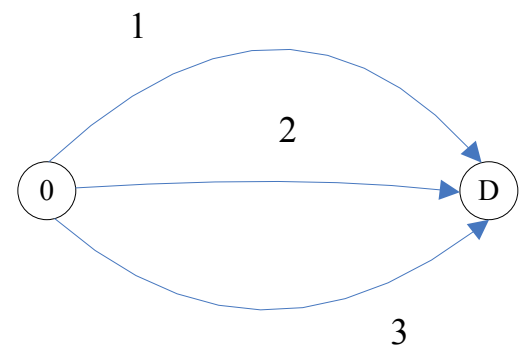

Fig. 2.1 Series structure
Fig. 2.2 Parallel strucure

Fig. (2). The basic structure of road network. 
from uncertain dynamic network to a specific state network for each stage.

The process order for road rehabilitation is the first to settle. The priority for road repair is the most important sections in the road network to the earthquake relief. If a road is interrupted not to delivery to the affected points (namely, the post-earthquake road connectivity is 0 ), the section should be priority to repair. If multiple sections is interrupted not to access to the affected points, the section, to the demand urgent affected point connectivity is 0 , need to be rehabilitated prior. If the connectivity to access to one certain affect point is 0 owing to multiple sections interrupted, the priority should be to the path to the affected as soon as possible. If the accessibility has no effect due to road sections interrupted, the section, the max of weighted connectivity, should be rehabilitated prior. The expression of weighted connectivity is as below.

$$
E_{o d}^{\prime}=\sum_{d \in D} \sum_{w \in W} w_{d} \cdot q_{d m} \cdot\left(1 / t_{o d}\right) \cdot E_{o d}
$$

\subsubsection{Single Stage Static Problem}

The post-earthquake rescue phase usually can be divided into two stages, emergency period and continued to rescue. The emergency rescue stage occurred in the 72 hours after the earthquake, also known as golden relief period, and continuing rescue period continue for several days according to the damage caused by the earthquake. From the perspective of emergency material distribution, emergency period should give priority to fairness considering efficiency; and continuing rescue period is giving priority to efficiency considering fairness. In the model formulation, the objective function should balance between fairness and efficiency. Efficiency can be achieved by the total delay time minimum and the total delivery time minimum, and fairness is reflected by the total weighted distribution volume unmet considering demand urgence for affected points.

Mathematical model is established as follow.

$$
\begin{aligned}
& \min Z=\sum_{d \in D} \sum_{m \in M} w_{d}\left(Q_{d m}-\sum_{d \in D} S_{k d m} N_{k d m}\right)+ \\
& \alpha \sum_{k \in K} \sum_{d \in D} \sum_{m \in M} \Delta t_{k d m} \Delta S_{t d m} N_{k d m}+\beta \sum_{k \in K} \sum_{d \in D} \sum_{m \in M} 2 t_{k d m} S_{k d m} N_{t d m} \\
& \text { s.t. } \sum_{d \in D} \sum_{k \in K} y_{o d k} \leq n \\
& \sum_{i \in c} y_{o i k}-\sum_{i \in c} y_{o i k}=0, \forall k \in K \\
& \sum_{j \in C} x_{i j k}-\sum_{j \in C} x_{j i k}=0, \forall i \in I, \forall k \in K \\
& \sum_{d \in D} S_{t d m} N_{k d m} \leq Q_{d m}, \forall d \in D, \forall m \in M \\
& T_{j k}=T_{i k}+x_{i j k} \delta_{i j k}, \forall i \in C, \forall j \in C, \forall k \in K \\
& x_{i j k}\left(T_{j k}-T_{i k}\right) \geq 0, \forall i \in C, \forall j \in C, \forall k \in K
\end{aligned}
$$

The objective function can be achieved optimization for the emergency period and the continuous period by adjusting the coefficients $\alpha$ and $\beta$. Usually, the emergency rescue stage, $\alpha$ and $\beta$ can be proper small, and continuous relief period, appropriate bigger.

In the constraint conditions, the formula (7) represents restriction of the total distribution vehicle; the formula (8) expresses the vehicle routes starting from the emergency rescue center to the affected points and then returning the emergency rescue center; the formula (9) restricts the consistent in access and exit one point; the formula (10) represents the meaning that distribution materials are no more than the total amount of demand for any affected point; the formula (11) expresses the time when the vehicle arrives the network node; the formula (12) ensure the vehicle order from $i$ to $j$.

\section{MODEL METHODOLOGY}

As shown in Fig. (3), multi stage dynamic problem is transformed the each stage state optimization problem of the road rehabilitation and emergency material distribution. Each stage is divided by the network changes, and the order for road rehabilitation depends on the content of 3.2.1 section.

For each stage problem of post-earthquake road rehabilitation and emergency material distribution, the simulated annealing algorithm is proposed to solve it.

Step 1. The minimization objective function without constraints is structured through the penalty function to the initial objective function.

$\min \mathrm{F}=Z+M \cdot P$

Among them, $Z$ represents the initial objective function, and $M$ is an infinite number. $P$ can express the formula (14) as follow.

$$
\begin{aligned}
& P=\left\{\max \left[\left(\sum_{d \in D} \sum_{k \in K} y_{o d k}-n\right), 0\right]\right\}^{2}+\max \left[\left(\sum_{i \in c} y_{o i k}-\sum_{i \in c} y_{o i k}\right)^{2}, o\right] \\
& +\max \left[\left(\sum_{j \in C} x_{i j k}-\sum_{j \in C} x_{j i k}\right)^{2}, o\right]+\left\{\max \left[\left(\sum_{d \in D} S_{t d m} N_{k d m}-Q_{d m}\right), 0\right]\right\}^{2} \\
& +\max \left[\left(T_{i k}+x_{i j k} \delta_{i j k}-T_{j k}\right)^{2}, o\right]+\left\{\min \left[x_{i j k}\left(T_{j k}-T_{i k}\right), 0\right]\right\}^{2}
\end{aligned}
$$

Step 2. $k=0$ is given. The initial solutions are given for $x_{i j k}$ and $y_{o d k}$, denoted as $x_{i j k}^{0}$ and $y_{o d k}^{0}$. The selected initial temperature is set to $T_{0}>0$, and $F\left(x_{i j k}^{0}, y_{o d k}^{0}\right)$ is calculated. $S_{\min }=\left(x_{i j k}^{0}, y_{o d k}^{0}\right)$ and $F_{\min }=F\left(x_{i j k}^{0}, y_{o d k}^{0}\right)$ are given.

Step 3. New candidate solutions are generated by the initial solution $x_{i j k}$ and $y_{o d k}$, according to the formula " $x_{i j k}^{S+1}=x_{i j k}^{S}+r g$ and $y_{o d k}^{S+1}=y_{o d k}^{S}+a l$ ", in which, $r$ and $a$ are random variables, 0 to $1, g$ and $l$ are the adjusting factors in the form of linear attenuation function. 


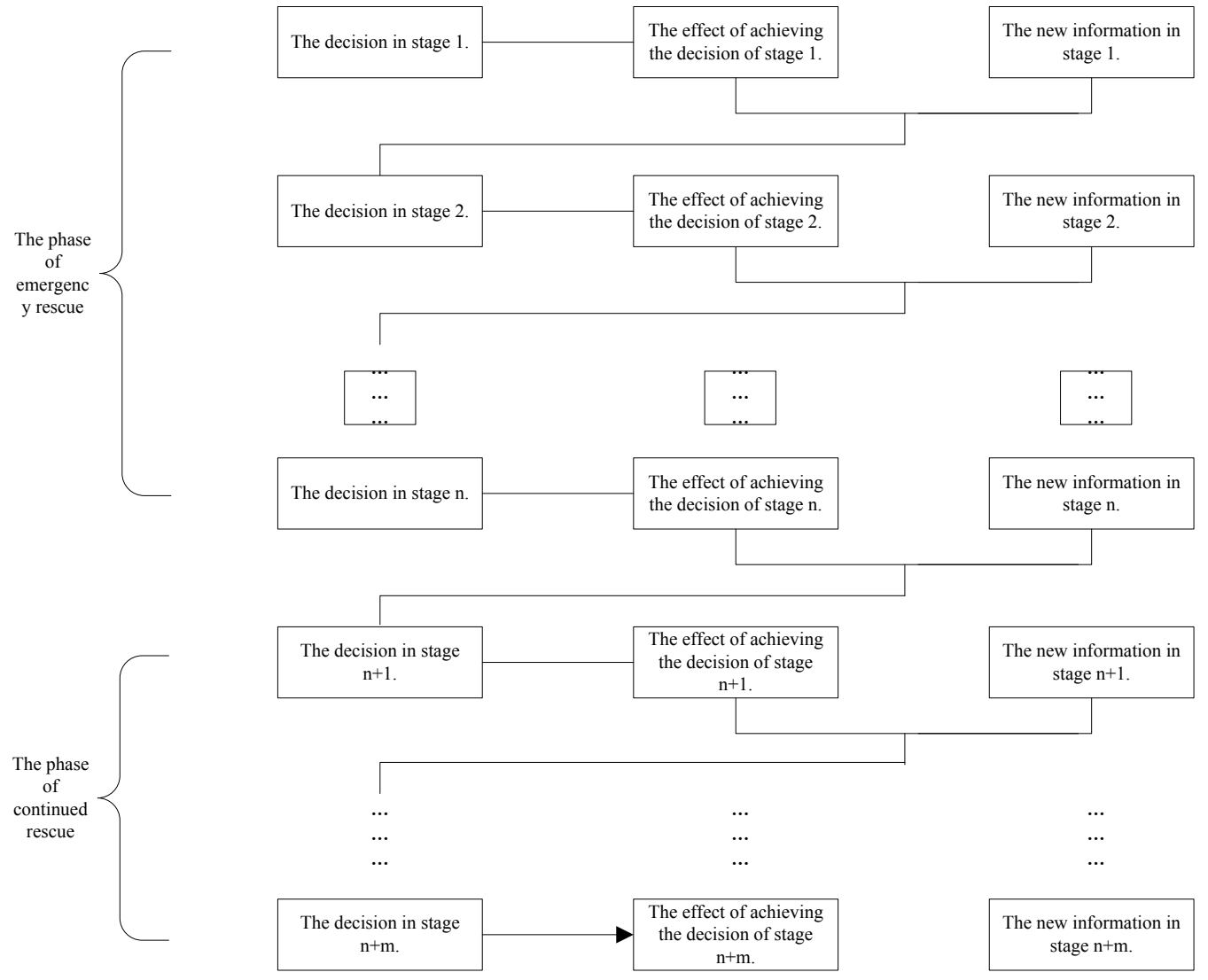

Fig. (3). Schematic diagram of multi stage dynamic problem solving.

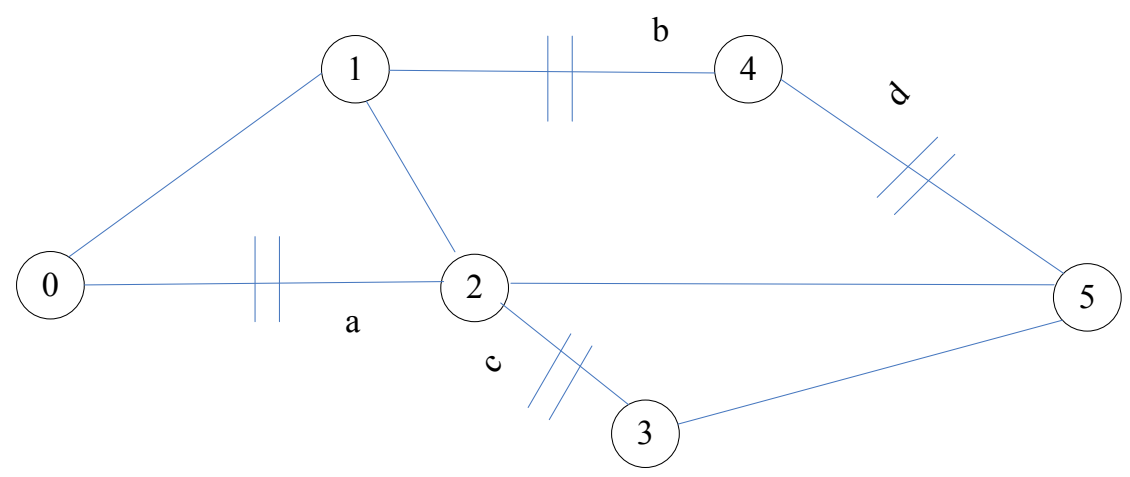

Fig. (4). The road network of numerical example.

Step 4. $\Delta F=F\left(x_{i j k}^{k+1}, y_{o d k}^{k}\right)-F\left(x_{i j k}^{k}, y_{o d k}^{k}\right)$ is calculated. If $\Delta F \leq 0$, the new solutions are accepted, or a random variable $\xi$ is generated, and if $e^{-\Delta f / T}>\xi$, the new solutions are accepted by the probability of $e^{-\Delta f / T}$.

Step 5. If $F^{k+1}<F_{\min }, \quad S_{\min }=S^{k+1}, \quad F_{\min }=F^{k+1}$

Step 6. If the termination is satisfied, the algorithm terminates, thus $S_{\min }$ is as an approximation optimal solution, and $F_{\min }$ as the corresponding optimal value. Otherwise, continue to the step 7 .
Step 7. A new temperature is generated $T_{k+1}$ according to the existing temperature, and $k=k+1$ is given. Then turn to the step 2.

\section{NUMERICAL SIMULATION}

As is shown in the Fig. (4), four sections $a, b, c, d$, are interrupted by the earthquake. They are assumed 5 affected points and 20,50,45, 40 units as the rehabilitation time for each damaged road. Emergency material distribution can only adopt the form of ground of highway transportation. 
Table 1. Relative parameters of different demand points.

\begin{tabular}{|c|c|c|c|c|c|}
\hline Demand Points (Affected Points) & 1 & 2 & 3 & 4 & 5 \\
\hline Demand quantity & 40 & 40 & 80 & 40 & 80 \\
\hline Demand urgent coefficient & 0.1 & 0.15 & 0.25 & 0.4 & 0.1 \\
\hline Latest delivery time & 140 & 120 & 110 & 90 & 140 \\
\hline
\end{tabular}

Table 2. Unmet distribution amount, delayed time and distribution routes for different stages.

\begin{tabular}{|c|c|c|c|c|c|c|}
\hline \multirow{4}{*}{$\begin{array}{c}\text { The } 1^{\text {st }} \text { stage } \\
\text { (no completed sections) }\end{array}$} & Unmet distribution amount & 40 & 20 & 40 & 40 & 80 \\
\hline & Delayed time & 0 & 0 & 0 & 0 & 0 \\
\hline & Distribution routes for the $1^{\text {st }}$ vehicle & \multicolumn{5}{|c|}{ In order delivery to the demand point of 3 and 1 , then return to the point 0} \\
\hline & Distribution routes for the $2^{\text {nd }}$ vehicle & \multicolumn{5}{|c|}{ In order delivery to the demand point of 3 and 2 , and stay at the demand point 2} \\
\hline \multirow{4}{*}{$\begin{array}{c}\text { The } 2^{\text {nd }} \text { stage (section b } \\
\text { completed) }\end{array}$} & Unmet distribution amount & 20 & 20 & 40 & 20 & 60 \\
\hline & Delayed time & 0 & 0 & 0 & 0 & 0 \\
\hline & Distribution routes for the $1^{\text {st }}$ vehicle & \multicolumn{5}{|c|}{ Delivery to the demand point 4 , and return to the point 0} \\
\hline & Distribution routes for the $2^{\text {nd }}$ vehicle & \multicolumn{5}{|c|}{ Delivery to the demand point 5 , and return to the point 0} \\
\hline \multirow{4}{*}{$\begin{array}{c}\text { The } 3^{\text {rd }} \text { stage (section a } \\
\text { completed) }\end{array}$} & Unmet distribution amount & 20 & 0 & 0 & 0 & 40 \\
\hline & Delayed time & 0 & 0 & 5 & 0 & 0 \\
\hline & Distribution routes for the $1^{\text {st }}$ vehicle & \multicolumn{5}{|c|}{ Delivery to the demand point 4 twice, and return to the demand point 3} \\
\hline & Distribution routes for the $2^{\text {nd }}$ vehicle & \multicolumn{5}{|c|}{ In order delivery to the demand point of 4,5 and 2 , and stay at the point 2} \\
\hline \multirow{4}{*}{$\begin{array}{c}\text { The } 4^{\text {th }} \text { stage (section c } \\
\text { completed) }\end{array}$} & Unmet distribution amount & 0 & 0 & 0 & 0 & 0 \\
\hline & Delayed time & 0 & 0 & 0 & 0 & 10 \\
\hline & Distribution routes for the $1^{\text {st }}$ vehicle & \multicolumn{5}{|c|}{ Delivery to the demand point 5 , and return to the point 0} \\
\hline & Distribution routes for the $2^{\text {nd }}$ vehicle & \multicolumn{5}{|c|}{ In order delivery to the demand point of 1 and 5 , then return to the point 0} \\
\hline
\end{tabular}

No new sections are interrupted, in the rescue phase, and namely the road network structure changes only caused by the initial earthquake. For the example, following contents are assumed: emergency rescue center raise materials 320 units, and supplies a constant number in the condition of no complementary again; the vehicle number for distribution is 2 , and the maximum carrying capacity is 20 units; the running time for all section is 5 units; the demand quantity and the latest delivery time requirements are shown in the Table 1.

Some parameters for simulated annealing algorithm are as below: the initial maximum temperature of 1000 , minimum temperature of 0.1 , the attenuation coefficient of 0.99 , and Markov chain length of 15 .

Through the simulation, the damaged road rehabilitation order is bacd, and unmet distribution amount, delayed time and distribution routes for different stages are as shown in the following Table 2.

\section{CONCLUSION}

The road network damaged by the earthquake, need to be carried out to repair. Due to the aftershocks and secondary disasters, and partial roads rehabilitation completed, it dynamically changes to the road network, relief emergency distribution relied on. In order to study on the problem of the collaborative optimization of Emergency Supply and Road Rehabilitation, The paper has done the following work. Firstly, the post-earthquake road network connectivity is defined, and the rehabilitation order is solved by the extended meaning of connectivity. Secondly, the multistage dynamic problem is decomposed to multiple single phase problems. For each single one, the mathematical model, which balances between efficiency and fairness, is established, and a simulated annealing algorithm is adopted for solving the model. Last, a numerical example was adopted to verify the method feasibility. The next step of research can enrich the connotation of theoretical research through access to the actual data, to make it more realistic significance. 


\section{CONFLICT OF INTEREST}

The authors confirm that this article content has no conflict of interest.

\section{ACKNOWLEDGEMENTS}

This work is supported by the Teachers Scientific Research Fund of China Earthquake Administration (No. 20140107).

\section{REFERENCES}

[1] F. Fiedrich, F. Gehbauer, and U. Rickers, "Optimized resource allocation for emergency response after earthquake disasters", Safety Science, vol. 35, no. 1-3, pp. 41-57, 2000.

[2] Y. Sun, H. Chi, and C. Jia. "Mixed integer programming model for the emergency resource scheduling in multi-path", Operations Research and Management. vol. 16, no. 5, pp. 5-8, 2007.

[3] L. Ozdamar, E. Ekinci, and B. Kn9nkyazici, "Emergency logistics planning in natural disasters" Annals of Operations Research, vol. 129, no. 14, pp. 217-245, 2004.

[4] G. Barbarosoglu, and Y. Arda, "A two-stage stochastic programming framework for transportation planning in disaster response", Journal of Me Operational Research Society, vol. 55, no. 1, pp. 4353, 2004.

[5] C.F. Hsuek, H.K. Chen, and H.W. Chou, "Vehicle Routing for Relief Logistics in Natural Disasters," D. Thesis, Jungli, Taiwan, 2007.

[6] S. Wang, "Research on Earthquake Network Repair Scheduling and the Endure Degree on the Repair Time in Imprecise Information", D. Thesis, Taipei, Taiwan, 2008.
[7] S. Yan, and Y. L. Shih, "A time-space network model for work team scheduling after a major disaster", Journal of the Chinese Institute of Engineers, vol. 30, no. 1, pp. 63-75, 2007.

[8] S. Yan, and Y. L. Shih, "Optimal scheduling of emergency roadway repair and subsequent relief distribution", Computers \& Operations Research, vol. 36, no. 6, pp. 2049-2065, 2008.

[9] R. Guo, "The shortest path problem of material transportation in interval time", Journal of Beijing Institute of Technology (Social Science Edition), vol. 8, no. 8, pp. 29-30, 2006.

[10] Y. Shi, "Research on Logistics Operation Scheduling and Earthquake Engineering Repair After Earthquake", D. Thesis, Taipei, Taiwan, 2004.

[11] Y. Zhang, X. Guo, and J. Li, "The integrated optimization algorithm of post disaster road repair and material distribution", Journal of Traffic and Transportation Engineering, vol. 7, no. 2, pp. 117-122, 2007.

[12] A. Li, "Research on the Scheduling of Emergency Road Repair and Relief Ristribution Rost Earthquake", Ms, Thesis, Chengdu, China, 2010 .

[13] H. K. Liao, “A Study on Network Reconstruction and Relief Logistics" D. Thesis, Taipei, Taiwan, 2005.

[14] S. Chen, and J. Jiang "Emergency logistics distribution problem model under uncertain roadway network structure and its application", Systems Engineering-Theory \& Practice, vol. 31, no. 5, pp. 907-913, 2011.

[15] L. Zheng, "Combined Optimization of Post-Earthquake Relief Relive and Road Rehabilitation" Ms, Thesis, Chengdu, China, 2012.

[16] S. Chen, "Research on Emergency Resources Scheduling Problem with Variable Road Network Structure" D. Thesis, Changsha, China, 2011 